\title{
Technè
}

La science au service de l'histoire de l'art et de la préservation des biens culturels

$41 \mid 2015$

Arts textiles antiques et modernes. Approche scientifique et restauration

\section{Restauration d'un linceul égyptien d'époque romaine pour les nouvelles salles du musée du Louvre}

Restoration of an Egyptian shroud dating from the Roman period for the new galleries at the Louvre

Florence Gombert-Meurice, Caroll Fierle, Patricia Dal-Prà, Noëlle Timbart et Laure de Guiran

\section{OpenEdition Journals}

Édition électronique

URL : http://journals.openedition.org/techne/4717

DOI : 10.4000/techne.4717

ISSN : 2534-5168

Éditeur

C2RMF

Édition imprimée

Date de publication : 25 novembre 2015

Pagination : 78-85

ISBN : 978-2-7118-6248-1

ISSN : $1254-7867$

Référence électronique

Florence Gombert-Meurice, Caroll Fierle, Patricia Dal-Prà, Noëlle Timbart et Laure de Guiran,

"Restauration d'un linceul égyptien d'époque romaine pour les nouvelles salles du musée du Louvre », Technè [En ligne], 41 | 2015, mis en ligne le 08 août 2020, consulté le 11 mars 2021. URL : http:// journals.openedition.org/techne/4717 ; DOI : https://doi.org/10.4000/techne.4717

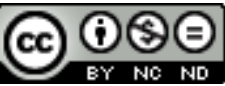

La revue Technè. La science au service de l'histoire de l'art et de la préservation des biens culturels est mise à disposition selon les termes de la Licence Creative Commons Attribution - Pas d'Utilisation Commerciale - Pas de Modification 4.0 International. 


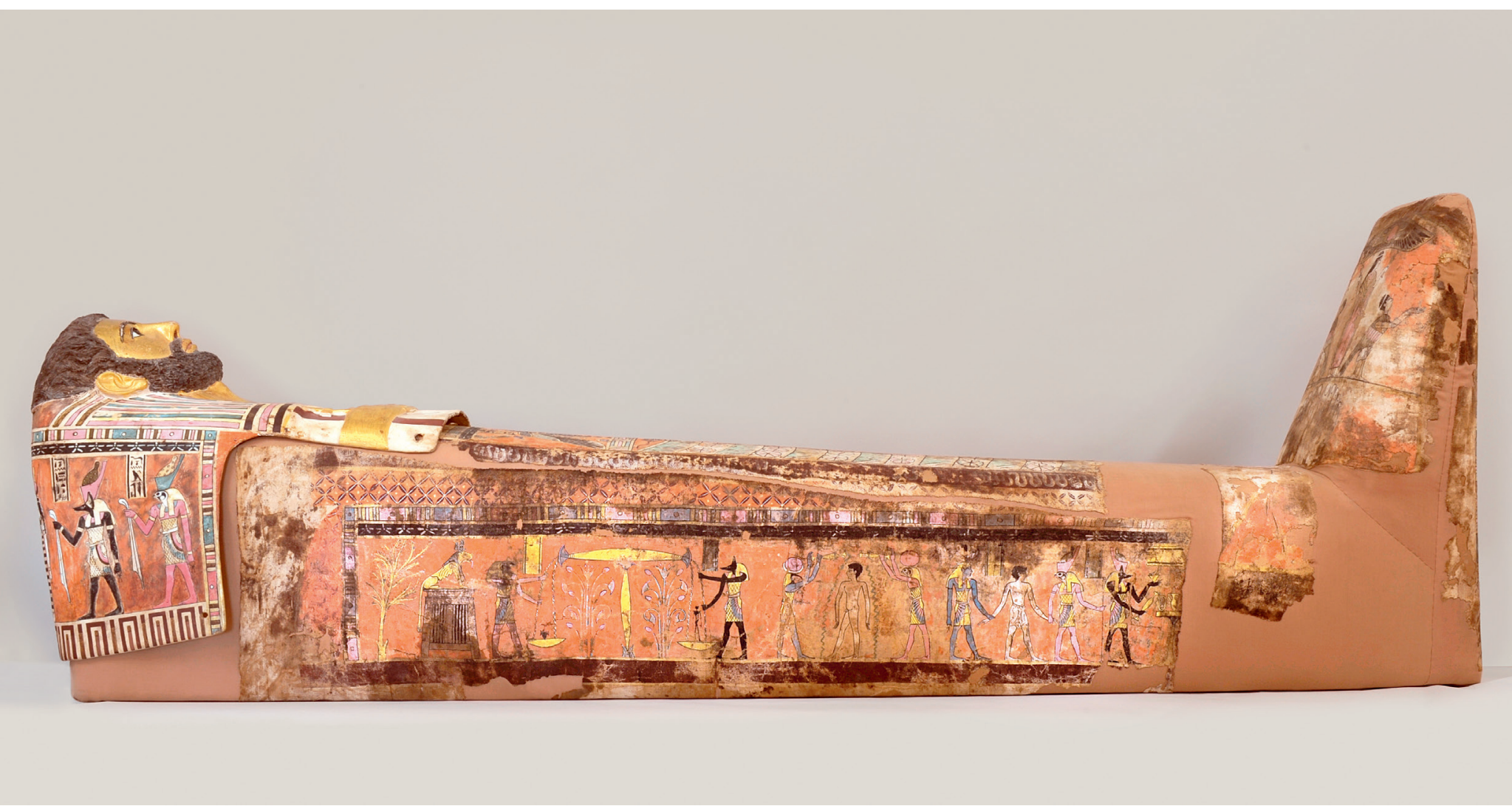

Fig. 1. Le linceul et son masque-plastron après intervention. () C2RMF/Anne Chauvet. 
Florence Gombert-Meurice

Caroll Fierle

Patricia Dal-Prà

Noëlle Timbart

Laure de Guiran

\section{Restauration d'un linceul égyptien d'époque romaine pour les nouvelles salles du musée du Louvre}

Restoration of an Egyptian shroud dating from the Roman period for the new galleries at the Louvre

\begin{abstract}
Résumé. L'ouverture des nouvelles salles consacrées à l'Orient romain au musée du Louvre a été l'occasion de restaurer un linceul daté du II siècle ap. J.-C. conservé au département des Antiquités égyptiennes avec son masque-plastron. La présentation de cet ensemble qui combine, fait assez rare dans un musée, le plastron avec son linceul d'origine, a supposé de réfléchir à la façon dont ce dernier était agencé sur la momie originale afin de lui restituer sa position la plus exacte possible. Une enquête historique pour trouver des linceuls comparables a, par conséquent, été nécessaire avant de procéder à la restauration proprement dite. Cette dernière a impliqué un travail préparatoire et la création d'un prototype pour ajuster la forme du montage aux fragments du linceul anciennement restaurés.

Mots-clés. Linceul romain, masque-plastron, remontage, textile, restauration, polychromie.
\end{abstract}

\begin{abstract}
The opening of new rooms reserved for "The East Mediterranean in the Roman Empire" at the Louvre provided an opportunity to restore a shroud dating from the 2 nd century $A D$, together with its mummy mask, on display in the Department of Egyptian Antiquities. The presentation of this ensemble in which the mummy mask is shown with its original shroud, quite a rare occurrence in museums, implied reflecting on the way in which the shroud was placed on the original mummy in order to reassemble it in an exact a position as possible. Consequently, historical research to find comparable shrouds was necessary before proceeding with the actual restoration. The latter involved preparatory work and the creation of a prototype in order to adjust the form of the assemblage to the fragments of the previously restored shroud.

Keywords. Roman shroud, mummy mask, reassemblage, textile, restoration, polychromy.
\end{abstract}

En 2000, trois ans après l'ouverture d'une galerie consacrée à l'art funéraire de l'Égypte romaine dans l'aile Denon du musée du Louvre, le département des Antiquités égyptiennes fit l'acquisition, par don de la Société des Amis du Louvre et de Lucien Viola, collectionneur ${ }^{1}$, d'un ensemble exceptionnel : un masque-plastron d'homme en stuc et le linceul de lin peint correspondant $^{2}$ (fig. 1). En 2011, le nouvel aménagement des mêmes salles pour la présentation de l'ensemble des collections de l'Orient romain ${ }^{3}$ fut l'occasion de reprendre le remontage de l'œuvre. La forme même de la structure de montage paraissait mal convenir et obligeait à de nombreuses superpositions de fragments de linceul, notamment au niveau des pieds, qui fragilisaient la couche picturale. Une nouvelle enquête historique était donc nécessaire, afin de procéder à une révision complète du montage.

\section{Enquête historique : la confirmation d'un modèle à suivre pour la restauration de l'œuvre}

L'enveloppe funéraire est composée d'un masque-plastron à dosseret qui couvrait la tête et le buste d'une momie, et d'un linceul peint aujourd'hui en plusieurs fragments. Le masqueplastron représente un homme barbu, le visage et les mains dorés, tenant dans sa main droite le symbole du souffle de vie. Sur le dosseret, Osiris-Sokaris est figuré entre les deux sœurs d'Osiris et diverses divinités lui tendent un fragment de lin, ce qui montre l'importance accordée à l'embaumement dans le processus de résurrection du défunt. Le linceul est couvert de scènes issues du Livre pour sortir le jour, se lisant de la tête aux pieds côté dextre (la pesée du cœur, la purification du défunt ensuite conduit devant Osiris) et des pieds à la tête côté senestre (le défunt sur son lit funéraire - en lacune -, suivi d'une théorie divine vers l'entrée de la chapelle funéraire sur laquelle figure le $b a$ (oiseau âme) du défunt, puis une

Florence Gombert-Meurice, conservateur du patrimoine, département des Antiquités égyptiennes, musée du Louvre (florence.gombertmeurice@louvre.fr). Caroll Fierle, restauratrice indépendante de peintures, conseil en conservation préventive (c.fierle@wanadoo.fr). Patricia Dal-Prà, restauratrice indépendante de textiles, conseil en conservation préventive (patricialdalpra@gmail.com).

Noëlle Timbart, conservateur du patrimoine, chargée des Antiquités égyptiennes et orientales, département Restauration, C2RMF (noelle.timbart@culture.gouv.fr).Laure de Guiran, restauratrice indépendante de sculptures (ldeguiran@wanadoo.fr). 
scène de rafraîchissement du défunt dans la mort grâce à l'eau tirée par le roi à l'aide d'un chadouf). Le dessus du linceul est couvert de symboles de protection et de renaissance (Horus Béhédety étendant ses ailes puis plusieurs frises de motifs végétaux). La scène sous les pieds n'est autre qu'une représentation d'Osiris auquel le défunt est identifié, alors que sur le dessus des pieds, le $b a$ du défunt représenté deux fois bénéficie d'une libation d'eau fraîche versée par une déesse.

Dans la première publication du linceul et du masque, Klaus Parlasca ${ }^{4}$ date ceux-ci de 125-135 ap. J.-C. en se fondant sur des critères stylistiques concernant la coiffure et la barbe du défunt. Il les rapproche en outre de deux momies, celle d'Anoubias $^{5}$ et celle d'Artemidora ${ }^{6}$ datées de la fin du I ${ }^{\text {er }}$ siècle ap. J.-C., provenant toutes deux de la nécropole de Meir (Moyenne-Égypte). Leurs masques-plastron à dosseret embồtant ont, comme celui du Louvre, les mains dénudées et en relief au niveau de la poitrine ; leur linceul est complet, mais simplement orné de motifs appliqués. L'auteur compare aussi le masque avec ceux acquis par le musée de Berlin en 1990 - de facture proche, très soignée - provenant une fois encore de Meir ${ }^{7}$.

Pourtant, au moment de l'acquisition de l'ensemble par le Louvre, Marie-France Aubert ${ }^{8}$, alors conservateur au département des Antiquités égyptiennes, propose comme lieu de provenance Hermopolis-Ouest (nécropole d'Hermopolis Magna/Touna el-Gebel, en Moyenne-Égypte), par comparaison avec le cercueil d'une dénommée Teüris précisément découvert dans cette nécropole et conservé au Allard Pierson Museum d'Amsterdam ${ }^{9}$.

En effet, les linceuls - plus ornés à l'époque romaine qu'aux périodes antérieures de l'histoire de l'Égypte - tiennent souvent lieu de dernière enveloppe, à la manière des cercueils auxquels ils peuvent donc leur être comparés, leurs fonctions étant de protéger le corps du défunt momifié et de constituer le « cocon» indispensable pour sa transfiguration en Osiris ${ }^{10}$.

Le parallélisme de cet ensemble avec le cercueil de Teüris se vérifie en de nombreux points :

1. La représentation du buste du défunt vêtu d'une tunique à clavi sur lequel les mains sont placées horizontalement en relief comme seules émergées de l'enveloppe funéraire (point commun avec les masques féminins de Meir) ;

2. Les proportions de l'étoffe autour du visage, descendant jusqu'aux clavicules - évoquant le némès d'Osiris pour notre masque $^{11}$ - dont la tête du défunt paraît sortir pour se redresser ;

3. La composition aérée et linéaire sur les côtés du sarcophage, comme sur les côtés du linceul, de scènes issues du Livre des morts;

4. Les pieds chaussés de sandales émergeant verticalement ${ }^{12}$; 5. La scène de libation du $b a$ du défunt (oiseau à tête humaine) par une déesse, sans doute Nout, placée au-dessus des pieds du défunt et comme dressée à la manière d'une stèle ;

6. Enfin, sous les pieds, au revers de la scène de libation du $b a$, la figure d'Osiris renaissant, debout, composée de la même manière dans les deux cas, bien qu'elle soit mieux conservée sur le cercueil, en particulier dans la partie supérieure, cintrée et ornée d'un Horus Béhédety comme une stèle.

Il fut alors décidé d'utiliser cette comparaison pour restituer plus fidèlement la forme originelle du linceul, et de suivre l'hypothèse d'une origine hermopolitaine, invitant à prévoir une hauteur importante pour les pieds et à défaire le remontage qui superposait le tissu à cet endroit ${ }^{13}$.

\section{Constat avant démontage du linceul}

Avant leur arrivée au Louvre, les sept fragments de toile peinte avaient été restaurés à New York en $1990^{14}$ : ils avaient été nettoyés, mis à plat, consolidés puis doublés au moyen d'adhésifs polyvinyliques sur une fine toile en coton et polyester de couleur assortie (fig. 2). Avant cette intervention, le linceul n'avait jamais été restauré et demeurait dans un état proche de celui dans lequel il se trouvait lors de sa découverte, mais malheureusement après avoir déjà été dégagé de sa momie (fig. 3). À son arrivée au Louvre, la toile de doublage américaine fut découpée autour des fragments afin d'être remontés sur une structure épousant la forme d'une momie. L'intervention ${ }^{15}$ consista à coller les fragments sur une toile de coton de couleur beige foncé (fig. 4), mise en forme sur une structure rigide et légère de nids d'abeille en carton, recouverte de feutre de polyester. La forme mesurait $62 \mathrm{~cm}$ de haut, $33 \mathrm{~cm}$ de large et $206 \mathrm{~cm}$ de long ; une autre structure amovible servait de support au masque.

En 2011, ce montage montrait des signes de faiblesse. Les fragments peints reprenaient la planéité donnée par la première restauration et se décollaient du tissu tendu sur le mannequin en forme de momie sur lequel ils étaient remontés depuis 2000. De plus, des fils de coton et de nylon avaient été rajoutés afin de les replaquer sur la forme, ce qui exerçait des pressions sur la couche picturale risquant de la cisailler. La toile peinte montrait aussi des pertes d'adhérence avec sa toile de doublage, pouvant engendrer des déformations irréversibles et des soulèvements de la couche picturale.

La forme même du mannequin posait des problèmes. En effet, le masque-plastron, réalisé par l'encollage de plusieurs toiles enduites sur lesquelles est façonné le visage en stuc, est légèrement cintré afin de s'ajuster sur le corps de la momie dans son linceul. Or le mannequin ne respectait pas ce cintrage : il était trop plat. Le masque-plastron était donc posé de manière instable et ne reposait qu'en bordure, ce qui entraînait des contraintes et accentuait le soulèvement du stuc le long d'une fissure située au niveau des mains. Ce soulèvement a été résorbé en 2011 par encollage des deux éléments, mais il était important de soutenir le plastron sur une forme cintrée pour éviter de nouvelles contraintes.

Les recherches historiques menées en vue de la restauration montrèrent en outre que le masque-plastron était placé trop en arrière par rapport au linceul et que le mannequin en forme de momie n'était pas assez incliné et beaucoup trop haut pour permettre l'ajustement du linceul : ce dernier ne 


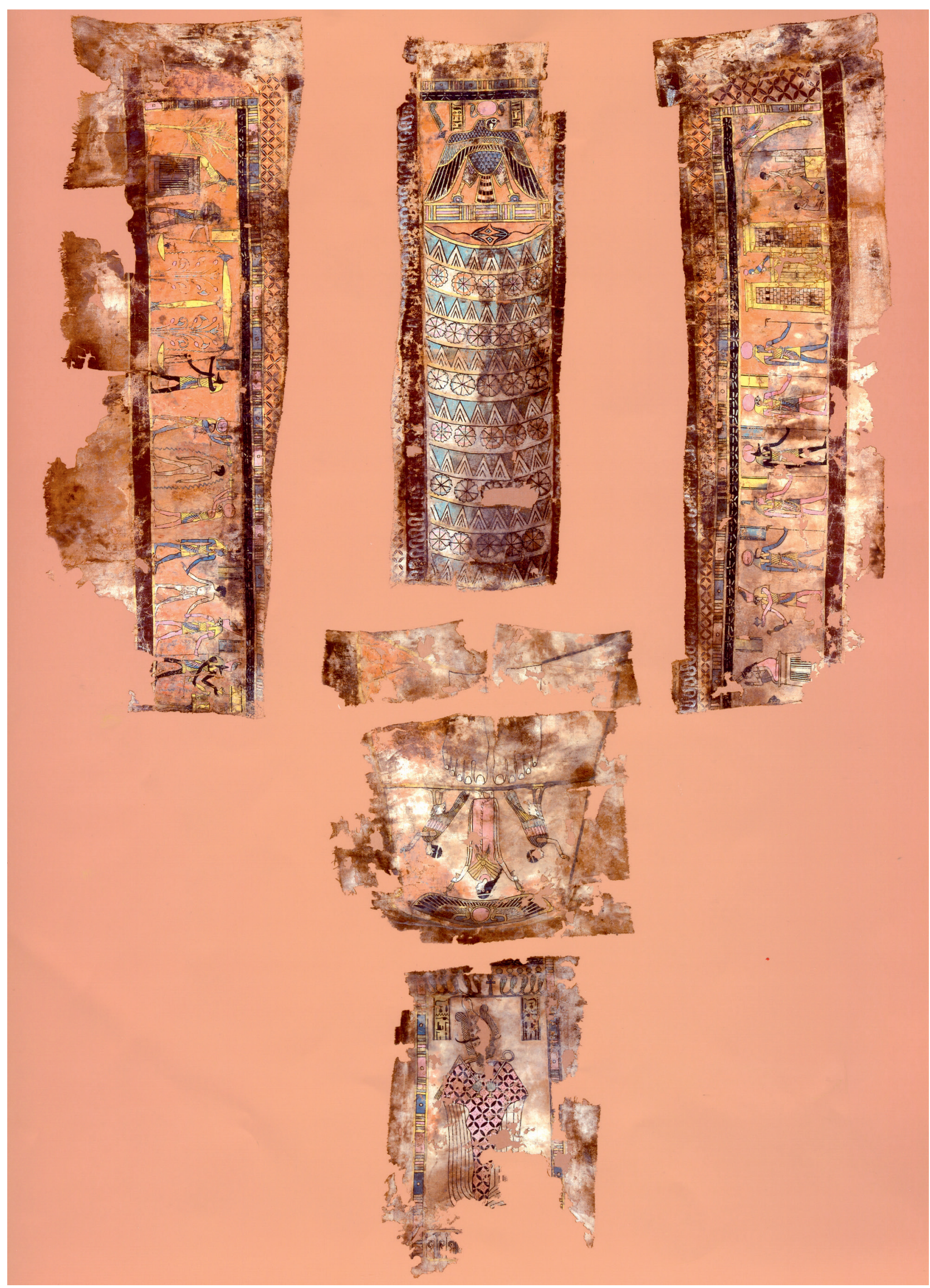

Fig. 2. Le linceul mis à plat. @ (Musée du Louvre/Georges Poncet. 

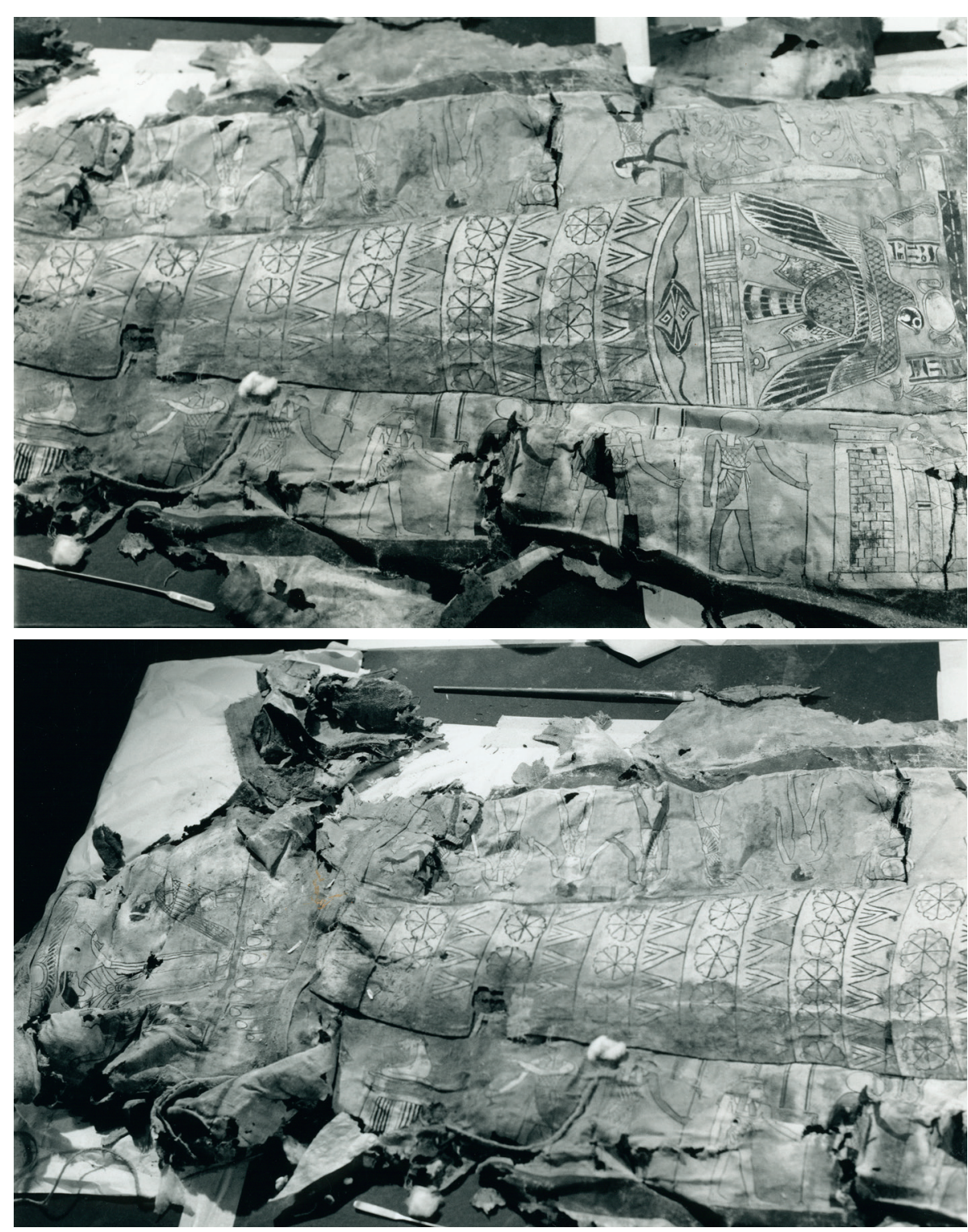

Fig. 3. Le linceul avant son traitement par Appelbaum et Himmelstein. Partie supérieure, en haut, inférieure, en bas. (C) Appelbaum \& Himmelstein.

suivait pas les motifs du plastron avec lesquels il devait s'aligner, notamment au niveau de la frise d'étoiles courant sur les côtés. Enfin, le raccord entre les pieds et le corps suivait une pente douce, très différente de celle du cercueil de Teüris qui suit un angle à presque $90^{\circ}$ et qu'il fallait manifestement davantage imiter.

L'étude mit aussi en évidence que certains fragments n'étaient pas correctement positionnés les uns par rapport aux autres. Par exemple, sur le côté dextre de la momie, au niveau des chevilles, la figure d'Anubis effectuant une libation était collée aux fragments des pieds et ne laissait pas l'espace suffisant pour imaginer la figure du dieu lui faisant face, probablement Osiris, aujourd'hui disparue. Côté senestre, il était également impossible d'imaginer la scène de déploration située au même niveau, dont seule la figure de Nephtys agenouillée ainsi que la tête du lion formant l'extrémité du lit du mort étaient conservées. Il était indispensable d'élargir l'espace à cet endroit pour redonner aux scènes toute leur place.

Une étude préalable à la restauration permit d'évaluer la possibilité d'une dérestauration, d'une restauration et d'un remontage ${ }^{16}$. Elle montra qu'il ne fallait pas tenter de décoller les fragments originaux de leur doublage de 1990, car ils étaient trop fragiles et cassants. De plus, leur adhésion était encore satisfaisante malgré quelques décollements localisés. Il paraissait en revanche possible d'envisager leur démontage de la forme réalisée en 2000 afin de les replacer sur une nouvelle structure. 


\section{Élaboration d'un prototype pour la forme et nouveau montage}

La restauration fut menée dans les ateliers de restauration du C2RMF situés dans le pavillon de Flore. Dans le même temps, une documentation scientifique par le biais de l'imagerie et des analyses non invasives par XRF furent réalisées pour identifier les pigments employés ${ }^{17}$. Après le retrait des fils de nylon qui maintenaient les fragments, ces derniers furent dégagés mécaniquement de la toile de coton qui était tendue sur la structure en forme de momie.

Un relevé des contours et des motifs de chaque fragment fut ensuite réalisé sur calque. Cette opération était indispensable pour les futures manipulations et les essais de positionnement. Il était ainsi possible d'assembler les calques de manière à reconstituer les motifs du linceul à plat sans utiliser les fragments originaux.

Ces calques furent photographiés et réduits par Hélène Guichard, conservateur en chef au département des Antiquités égyptiennes, afin d'être repositionnés selon diverses hypothèses sur une maquette à petite échelle épousant une forme de momie imaginée à partir du cercueil de Teüris (fig. 5). Un prototype à taille réelle fut ensuite réalisé pour disposer les calques grandeur nature et vérifier leurs emplacements (fig. 6). L'ancienne structure en forme de momie fut partiellement démontée puis taillée afin de la diminuer en hauteur, et de lui donner une pente plus importante. Elle fut ensuite recouverte avec de la ouate de polyester pour arrondir les arêtes. Un gabarit réalisé à partir de la courbure du masque permit finalement de réaliser le bombé de la forme de la momie.

Les scènes lacunaires côté dextre et senestre des pieds furent remontées en laissant davantage d'espace, sans toutefois reconstituer la longueur initiale pour des raisons esthétiques (les lacunes auraient été trop importantes) et techniques (les dimensions de la vitrine de présentation étant contraignantes).

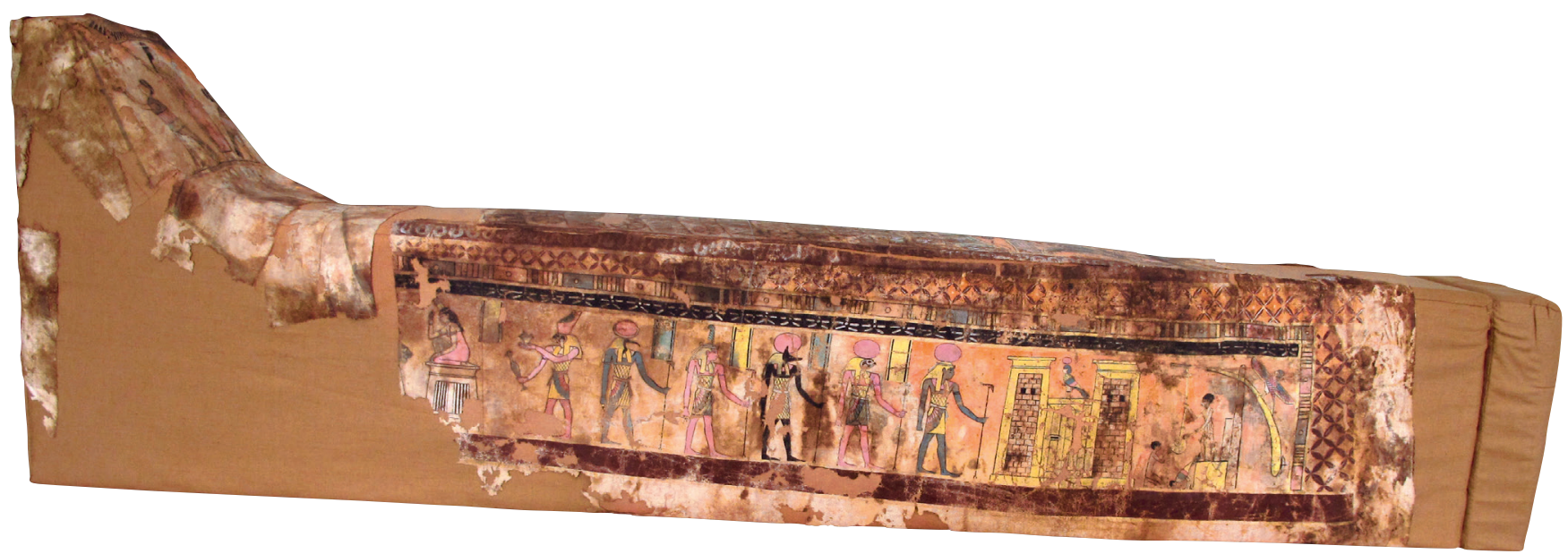

En outre, il fut décidé que la ligne brune de la partie inférieure du linceul servirait de base pour définir l'horizontalité de la forme, ce qui conduisait aussi à réduire le profil de la «momie » en hauteur.

Pour respecter la position initiale du plastron sur le linceul, il eut fallu recouvrir le motif du faucon situé au niveau de la poitrine. Toutefois, afin d'offrir aux visiteurs une meilleure vision de l'ensemble, il fut décidé de laisser le faucon apparent et de placer les bords latéraux du plastron à l'aplomb d'une frise verticale décorée d'étoiles peinte sur le linceul. Ainsi, seuls les motifs géométriques situés au-dessus du faucon furent cachés. Cette décision détermina la longueur totale de la forme, soit $215 \mathrm{~cm}$.

Il restait à fabriquer la structure en forme de momie définitive à partir du prototype grandeur nature réalisé, puis à y placer le linceul.

Des plaques rigides en mousse de polyéthylène furent découpées et collées ensemble avec un pistolet à colle à chaud. Elles furent taillées en escalier afin d'être plus aisément sculptées par la suite. Pour rigidifier la structure, une plaque de polypropylène cannelé fut collée en dessous, au pistolet à colle à chaud. La forme fut ensuite habillée avec de la ouate de polyester. L'ensemble fut revêtu d'une toile de lin de couleur beige. Une toile de coton fine, mais suffisamment solide, fut validée, puis tendre l'ensemble sur la forme. Après échantillonnage $^{18}$, cette toile fut teinte en harmonie avec la toile de consolidation utilisée en 1990 ponctuellement apparente. Elle fut mise à plat et découpée en deux parties, une pour le corps, et une autre pour les pieds, afin de l'ajuster convenablement à la forme. Il se révéla en effet impossible de tout replacer en une seule fois sans faire de plis. Ces contraintes, inhérentes à la forme originale du linceul, doivent sans doute inciter à réfléchir aux procédés de décoration du linceul. La la momie ? Cela signifie-t-il que la momie était déjà réalisée quand le linceul était peint? L'enquête doit aujourd'hui être menée. sélectionnée pour y coller les fragments dans leur position peinture était-elle réalisée une fois que celui-ci était placé sur

Fig. 4. Le linceul avant démontage. (C) Patricia Dal-Prà. 


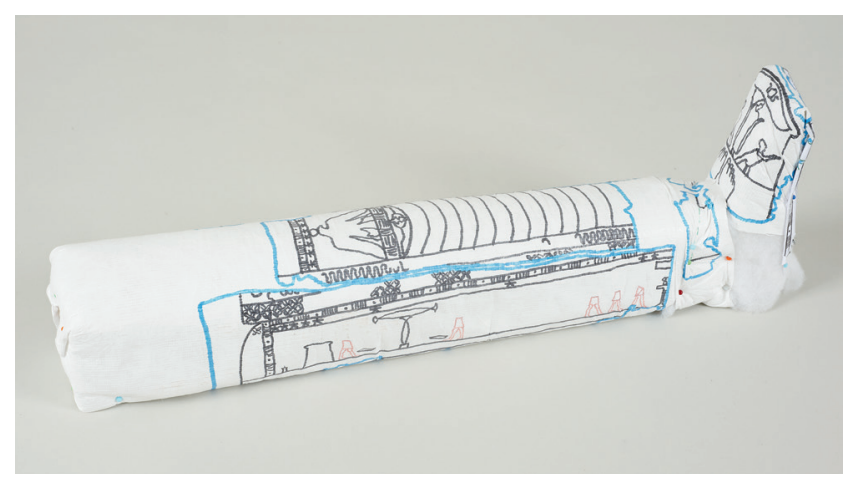

Préalablement à l'intervention de doublage, l'adhésion de tous les fragments peints à la toile de renfort « américaine » fut vérifiée. Suite aux différents pliages occasionnés par l'ancienne forme, certaines zones, essentiellement le long des bords, n'étaient plus adhérentes et nécessitaient des consolidations ${ }^{19}$. L'adhésion des petites poches de décollement au centre des fragments (où l'adhésif ne pouvait pas être inséré) fut reprise par régénération de l'ancien adhésif à la chaleur avec une spatule chauffante.

L'adhésif destiné à replacer le linceul sur la nouvelle toile fut appliqué au revers de chaque fragment et collé à la toile « américaine » par régénération à chaud. Les fragments furent positionnés très précisément sur les calques préalablement assemblés entre eux. L'intervention de collage se faisant par le revers, les calques furent inversés et la face des fragments placée au-dessus. La toile de coton teinte fut tendue sur un bâti de travail pour l'intervention de doublage afin de procéder au collage par régénération à chaud.

La toile fut ensuite fixée sur la structure en forme de momie par couture dans les zones sans couche picturale.
Fig. 5. Maquette à petite échelle imaginée à partir du cercueil de Teüris, pour aider à déterminer le positionnement des fragments de linceul. (c) C2RMF/Anne Chauvet.

Cette opération nécessitait d'intervenir à deux, la toile devait être tendue et ajustée pendant le travail de couture pour éviter les faux plis. Dans un second temps, le corps et les pieds furent raccordés par couture sur le montage. En raison de la fragilité de la couche picturale et de toile originale, il ne fut cependant pas possible de restituer la forme initiale de la partie supérieure des pieds qui devait être arrondie, car cela aurait contraint à plier la toile rendue trop rigide par la mise à plat de 1990. Le montage ainsi effectué permet une réversibilité totale (fig. 1).

\section{Conclusion}

Dès son acquisition par le musée du Louvre, la restauration de cette œuvre en 2000 avait abouti à une première restitution de l'ensemble du linceul et du masque d'homme suivant la forme qu'il devait avoir à l'origine lorsqu'il contenait la momie. Ce parti pris différait des présentations habituelles des linceuls d'époque romaine, exposés à plat. Ce choix se justifiait d'autant plus qu'il est très rare de pouvoir mettre un linceul et un masque-plastron en correspondance. La dernière restauration a permis de se rapprocher encore davantage de la forme originale et lance de nouvelles pistes de recherches sur la manière de concevoir et de décorer les linceuls qui venaient s'agencer sur des momies.

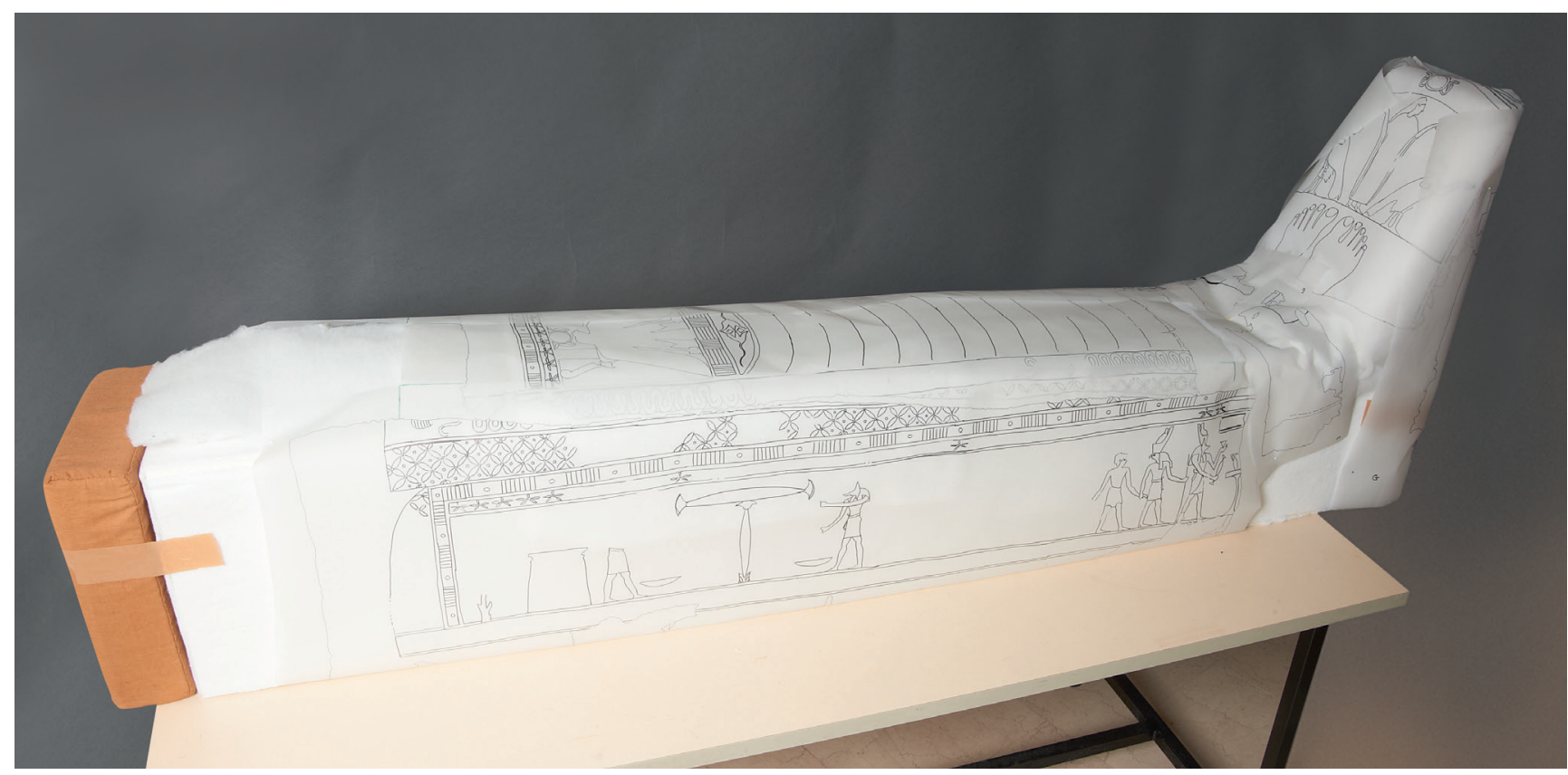

Fig. 6. Prototype réalisé pour disposer les calques grandeur nature. @ C2RMF/Anne Chauvet. 


\section{Notes}

Les auteurs tiennent à remercier Sandrine Pagès-Camagna, ingénieur de recherche au département Recherche du C2RMF pour sa collaboration à l'étude de cette enveloppe funéraire (analyses de la polychromie) et sa contribution à cet article.

1. Lucien Viola est un historien de l'art diplômé de l'Institute of Fine Arts de l'université de New York, spécialisé en art égyptien et en art contemporain. Il est également marchand, spécialisé dans l'art ancien et collectionneur. Dans les années 1970-1990, il était propriétaire de l'Ibis Gallery Ltd. à New York. Installé depuis les années 1990 à Marrakech, il forme des collections d'art marocain.

2. Aubert, 2000, p. 13-16. ; id., 2010, p. 18. Aubert et Cortopassi, 2008, cat. 12, p. 101-107.

3. Voir Gombert-Meurice, 2013, p. 36-60 ; Bel, Giroire et Gombert-Meurice, 2014, p. 66-70 ; id., 2012 b, p. 13-15.

4. Parlasca, $1999, \mathrm{n}^{\circ} 206$, p. 310.

Pour le linceul, voir Kurth, 1999, p. 92-97.

5. Elle est conservée au musée du Caire (CGC 33137, noté CGC 22137 par erreur dans la notice de K. Parlasca). Voir Edgar, $1905, n^{\circ} 33101-33285$, p. 32-34, pl. XVII.

6. Metropolitan Museum de New York, inv. 11.155.5. Voir Walker, 2000, $\mathrm{n}^{\circ} 85$, p. 132-134.

7. Voir Wildung, 1989 , p. 221-246. ; id., 1990, p. 206-221.

8. Aubert, 2000, p. 13-16.

9. Cercueil APM 7069. Voir Kurth, 1990. Sur l'ensemble, on pourra aussi consulter Bel, Giroire et Gombert-Meurice, 2012 a, p. 374-375 ; Derriks et Delvaux, 2009, p. 253 , note 7 pour les comparaisons iconographiques.

10. Pour la modalité de cette assimilation du défunt à Osiris, voir Smith, 2012, p. 187-196.

11. Notons d'ailleurs que les rayures du némès de notre masque sont très comparables à celles de masques en stuc plus fragmentaires conservés au musée du Caire et provenant précisément de Balansourah (Hermopolis-Ouest) comme le cercueil de Teüris. Voir Edgar, 1905, pl. XXIII.

12. Voir Scène de déploration du mort, d'après une tombe romaine d'Hermopolis, Gabra et Drioton, 1954, pl. 29.

13. Dans la nécropole, les momies sont d'ailleurs représentées suivant un schéma semblable, avec des pieds démesurément hauts. Voir à ce sujet et pour d'autres comparaisons, Riggs et Stadler, 2003, p. $69-87$, p. 80 , note 3 .

14. Information tirée du rapport de traitement rédigé par Barbara Appelbaum et Paul Himmelstein, dossier 89/55, 1990.

15. Intervention réalisée par MarieFrançoise de Rozières, alors restauratrice (relevant de la Direction des musées de France) auprès du département des Antiquités égyptiennes.

16. Dal-Prà, Fierle et Vincent, 2011 17. Pagès-Camagna, 2014.

L'identification de minium pour le décor de ce linceul confirme sa datation romaine. 18. Colorant Solophényl pour fibres cellulosiques fabriqué par Ciba Geigy, aujourd'hui Huntsman.

19. Insertion d'intissé collé au Beva ${ }^{\circledR}$ 371 film.

\section{Références bibliographiques}

Aubert M.-F., 2010, « Enveloppe de momie d'homme ", Grande Galerie, Le Journal du Louvre, 2000-2010, dix ans d'acquisition, Paris, p. 8.

Aubert M.-F., 2000, « Portrait d'un mort divinisé en Osiris Sokaris : don des amis du Louvre et de M. Lucien Viola au Louvre ", La Revue du Louvre et des Musées de France, n ${ }^{\circ}$ 5, p. 13-16.

Aubert M.-F. et Cortopassi R., 2008, Portraits funéraires de l'Égypte romaine. Cartonnages, linceuls et bois, RMN, Paris.

Bel N., Giroire C. et Gombert-Meurice Fl. (éd.), 2012 a, L'Orient romain et byzantin au Louvre, Actes Sud, Paris.

Bel N., Giroire C. et Gombert-Meurice Fl., 2012 b, « Les collections égyptiennes d'époque romaine du Louvre : nouvelle présentation ", Revue des Musées de France. Revue du Louvre, Paris, $\mathrm{n}^{\circ} 5$, p. 13-15.

Bel N., Giroire C. et Gombert-Meurice Fl., 2014, «L’Orient méditerranéen dans l'Empire romain ", La Recherche au musée du Louvre 2012, Paris, 2014, p. 66-70.

Derriks Cl. et Delvaux L. (éd.), 2009, Antiquités égyptiennes au Musée royal de Mariemont, Musée royal de Mariemont, Belgique.

Edgar C.C., 1905, Graeco-egyptian Coffins, Masks and Portraits, Catalogue général des antiquités égyptiennes du musée du Caire, IFAO, Le Caire.
Gabra S. et Drioton E., 1954, Peintures à fresques et scènes peintes à HermoupolisOuest (Touna el-Gebel), IFAO, Le Caire.

Gombert-Meurice Fl., 2013, « Les collections de l'Égypte romaine au Louvre ou les contours toujours redessinés d'une province de l'Empire ", BSFE, n ${ }^{\circ} 86-87$, p. 36-60.

Kurth D., 1990, Der Sarg der Teüris : Eine Studie zum Totenglauben im römerzeitlichen Ägypten, Aegyptiaca Treverensia, 6 , Mayence.

Kurth D., 1999, « Ein Weg zum ewigen Leben ", dans K. Parlasca et M.A. Stadler (dir.), Augenblicke. Mumienporträts und ägyptische Grabkunst aus römischer Zeit, Schirn Kunsthalle de Francfort, 30 janvier-11 avril 1999, Klinkhardt \& Biermann, p. 92-97.

Parlasca K. et Seemann H. (dir.), 1999, Augenblicke. Mumienporträts und ägyptische Grabkunst aus römischer Zeit, Schirn Kunsthalle de Francfort, 30 janvier-11 avril 1999, Klinkhardt \& Biermann.

Riggs Ch. et Stadler M. A., 2003, "A roman Shrouds and its demotic Inscriptions in the Museum of Fine Arts, Boston", JARCE, 40, p. 69-87.

Smith M., 2012, "New references to the Deceased as Wsir n NN from the Third intermediate period and the earliest Reference to the Deceased Woman as H.T-HR NN", Revue d'Égyptologie, 63, p. 187-196.

Walker S., (ed.), 2000, Ancient Faces: Mummy Portraits from Roman Egypt, New York, $\mathrm{n}^{\circ} 85$, p. $132-134$

Wildung D., 1990, «Geheimsvolle Gesichter ", Antike Welt, 21 Jahrgang, heft 4, p. 206-221.

Wildung D., 1989, «Mummienmasken aus Meir : Neuerwerbung des ägyptischen Museums ", Jahrbuch Preußischer Kulturbesitz, Band XXVI, XXX, p. 221-246.

\section{Documents inédits}

Appelbaum B. et Himmelstein P., 1990 "Mummy schroud, treatment report", Conservation of Works of Art, New York, dossier 89/55 du 13 juin 1990.

Dal-Prà P., Fierle C. et Vincent F., 2011, Rapport d'étude du linceul peint E32634 appartenant aux collections du musée du Louvre, décembre 2011.

Pagès-Camagna, S., Étude de la polychromie, Rapport de laboratoire, C2RMF, n²7522, 2014. 